\title{
Relationship Between Maximal Squat Strength and Five, Ten, ANd Forty Yard Sprint Times
}

\author{
Jeffrey M. McBride, Daniel Blow, Tyler J. Kirby, Tracie L. Haines, Andrea M. Dayne, \\ and N. Travis Triplett \\ Department of Health, Leisure, and Exercise Science, Neuromuscular Laboratory, Appalachian State University, Boone, \\ North Carolina 28608
}

\begin{abstract}
McBride, JM, Blow, D, Kirby, TJ, Haines, TL, Dayne AM, and Triplett, NT. Relationship between maximal squat strength and five, ten, and forty yard sprint times. J Strength Cond Res 23(6): 1633-1636, 2009-The purpose of this investigation was to examine the relationship between maximal squat strength and sprinting times. Seventeen Division I-AA male football athletes (height $=1.78 \pm 0.04 \mathrm{~m}$, body mass $[B M]=85.9 \pm 8.8 \mathrm{~kg}$, body mass index $[\mathrm{BMl}]=27.0 \pm 2.6 \mathrm{~kg} / \mathrm{m}^{2}, 1$ repetition maximum $[1 \mathrm{RM}]=166.5 \pm 34.1 \mathrm{~kg}, 1 \mathrm{RM} / \mathrm{BM}=1.94 \pm 0.33$ ) participated in this investigation. Height, weight, and squat strength (1RM) were assessed on day 1 . Within 1 week, 5, 10, and 40 yard sprint times were assessed. Squats were performed to a $70^{\circ}$ knee angle and values expressed relative to each subject's BM. Sprints were performed on a standard outdoor track surface with timing gates placed at the previously mentioned distances. Statistically significant $(p \leq 0.05)$ correlations were found between squat $1 \mathrm{RM} / \mathrm{BM}$ and 40 yard sprint times $(r=-0.605, p=0.010$, power $=0.747)$ and 10 yard sprint times $(r=-0.544, p=0.024$, power $=0.626)$. The correlation approached significance between 5 yard sprint times and 1RM/BM ( $r=-0.4502, p=0.0698$, power $=0.4421)$. Subjects were then divided into those above 1RM/BM of 2.10 and below $1 \mathrm{RM} / \mathrm{BM}$ of 1.90. Subjects with a $1 \mathrm{RM} / \mathrm{BM}$ above 2.10 had statistically significantly lower sprint times at 10 and 40 yards in comparison with those subjects with a 1RM/BM ratio below 1.90 . This investigation provides additional evidence of the possible importance of maximal squat strength relative to BM concerning sprinting capabilities in competitive athletes.
\end{abstract}

KEY WoRDS power, running, football, athletes

Address correspondence to Dr. Jeffrey M. McBride, mcbridejm@ appstate.edu.

23(6)/1633-1636

Journal of Strength and Conditioning Research

(C) 2009 National Strength and Conditioning Association

\section{INTRODUCTION}

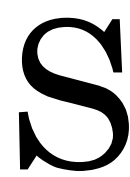
everal investigations have examined the possible relationship between sprinting ability and various strength measures $(1-5,7,9,10,13,15)$. This interest is a result of the defined relationship between the ability to apply a given value of ground reaction force and subsequent running velocity $(11,12,14)$. Several investigations have found a strong correlation between ground reaction force or impulse magnitude and sprinting velocity $(6,12)$. Some investigations have indicated smaller relationships between leg strength and sprinting velocity when measured in isolated single-joint movements such as a leg extension in either an isometric or isokinetic condition $(3,4,15)$, whereas structural multiple-joint measures of strength have been reported to have a stronger relationship to sprinting velocity (13).

Wisloff et al. (13) reported a -0.940 correlation between 10 meter sprint time and 1 repetition maximum (1RM) in a free weight squat. Dowson et al. (4) reported that concentric knee extension torque at $4.19 \mathrm{rad} / \mathrm{s}$ and time to reach 15 and 35 meters was significantly correlated at -0.518 and -0.688 , respectively. In an isometric leg extension, time to reach $60 \%$ of maximum force was significantly correlated to maximum running velocity $(r=-0.730)$ (3). Harris et al. (5) reported a statistically nonsignificant correlation between a machine squat $1 \mathrm{RM}$ and 10 meter and 40 meter sprint times $(r=$ $0.200,-0.140)$. Kukolj et al. (7) reported no significant correlations between isometric peak force of knee extensors, hip extensors and hip flexors, and any sprinting performance variables. Thus, it appears that a free weight multiple-joint structural measure of strength might have a more significant predictive capability in terms of sprinting ability.

Several investigations have shown the relationship between ground reaction force capabilities and sprinting performance $(6,11,12)$. A statistically significant correlation has been reported between maximum ground reaction force and maximal sprinting velocity $(r=0.600)(12)$. In addition, this investigation reported that maximal sprinting velocity was a product of ground reaction force and not the leg speed of the runners (12). Swing time for the legs of slow and fast runners was identical at approximately 0.360 seconds (12). In addition, Hunter et al. (6) has reported a significant 
correlation between ground reaction force horizontal impulse and sprinting velocity $(r=0.780)$. Thus, it is clear that strength or maximal force production is an integral component to maximal sprinting velocity.

Given the known relationship between ground reaction forces and sprinting velocity and the contribution of this ground reaction force from the major muscle groups of the lower body, it would appear as plausible that a $1 \mathrm{RM}$ in the squat might correlate to sprinting performance. Thus, this study attempted to further investigate the possible relationship between a structural multiple-joint assessment of strength (i.e., free weight squat) and its possible relationship to sprinting times.

\section{Methods}

\section{Experimental Approach to the Problem}

This study was designed to assess the possible relationship between maximal squat strength and sprint times at 5,10 , and 40 yards. Maximal strength of the lower-body musculature is required for maximal ground reaction forces that have been associated with maximal sprinting velocities. Thus, a 1RM squat and sprint times were measured and Pearson product correlation coefficients calculated between selected variables.

\section{Subjects}

Seventeen Division I-AA male football athletes (height $=1.78$ $\pm 0.04 \mathrm{~m}$, body mass $[\mathrm{BM}]=85.9 \pm 8.8 \mathrm{~kg}$, body mass index $[\mathrm{BMI}]=27.0 \pm 2.6 \mathrm{~kg} / \mathrm{m}^{2}, 1 \mathrm{RM}=166.5 \pm 34.1 \mathrm{~kg}$, $1 \mathrm{RM} / \mathrm{BM}=1.94 \pm 0.33$ ) participated in this investigation. All subjects voluntarily read and signed an informed consent, which was approved by the Institutional Review Board of Appalachian State University.

\section{Maximal Strength Testing}

Back squat 1RM was assessed after an appropriate warm-up protocol (8). The warm-up protocol consisted of multiple repetitions at loads equal to $30 \%$ ( $8-10$ repetitions), $50 \%$ (4-6 repetitions), $70 \%$ (2-4 repetitions), and 90\% (1 repetition) of the subject's estimated 1RM. During all attempts, subjects were required to lower the bar to a point where a $70^{\circ}$ knee angle was attained. Before the start of the warm-up, a goniometer was used to visually demonstrate the attainment of an $70^{\circ}$ knee angle while the subject was squatting. Subjects were given up to 4 maximal attempts to achieve a 1RM. Rest periods of 3 to 5 minutes were given between trials.

\section{Sprinting Times}

Sprint times of the 40 yard dash as well as 5 yard and 10 yard splits were measured using an infrared timing system (Brower Timing Systems, Draper, UT) performed on a standard track surface. Subjects activated the system by placing the thumb of their down hand on a touch-sensitive pad. Upon activation (initiated by thumb release from the touch-sensitive pad), the equipment emitted an auditory signal assuring the subject that he could begin his sprint at any time. In addition to the

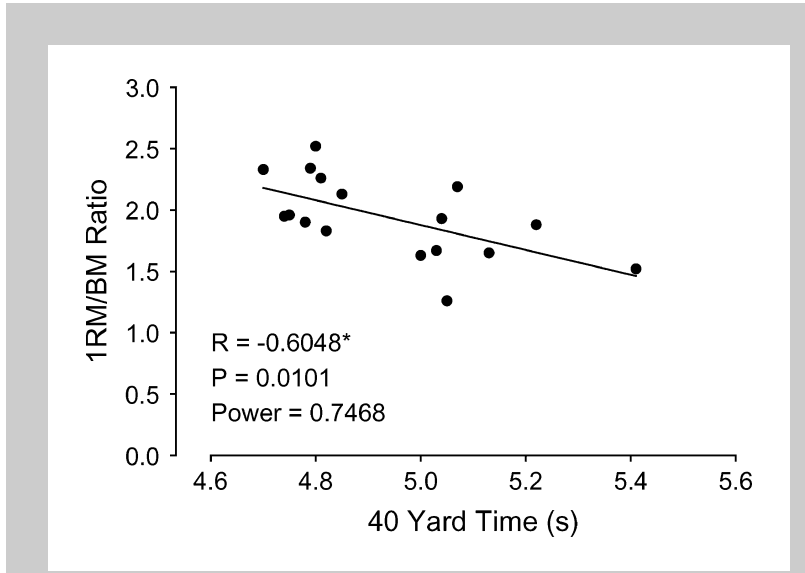

Figure 1. Relationship between 1 repetition maximum/body mass (1RM/ $\mathrm{BM}$ ) ratio and 40 yard sprint times.

assistance given by the equipment, the researcher was present at every start to ensure that the subject was lined up in a 3point stance in the correct spot and that he properly activated the system. No assistance such as running or starting technique was given during the study, nor was any encouragement given. The subject was instructed to run as fast as possible during the test and to make sure to run all the way through the clearly marked finish line. Reliability data for this test is reported by McBride et al. (8).

\section{Statistical Analyses}

Statistical analysis was performed using SPSS software (version 14.0, SPSS, Inc., IL,). Independent $t$-tests were used for mean comparisons between strong and weak groups. Pearson correlations were performed to determine significance between variables. The significance level was set at $p \leq 0.05$.

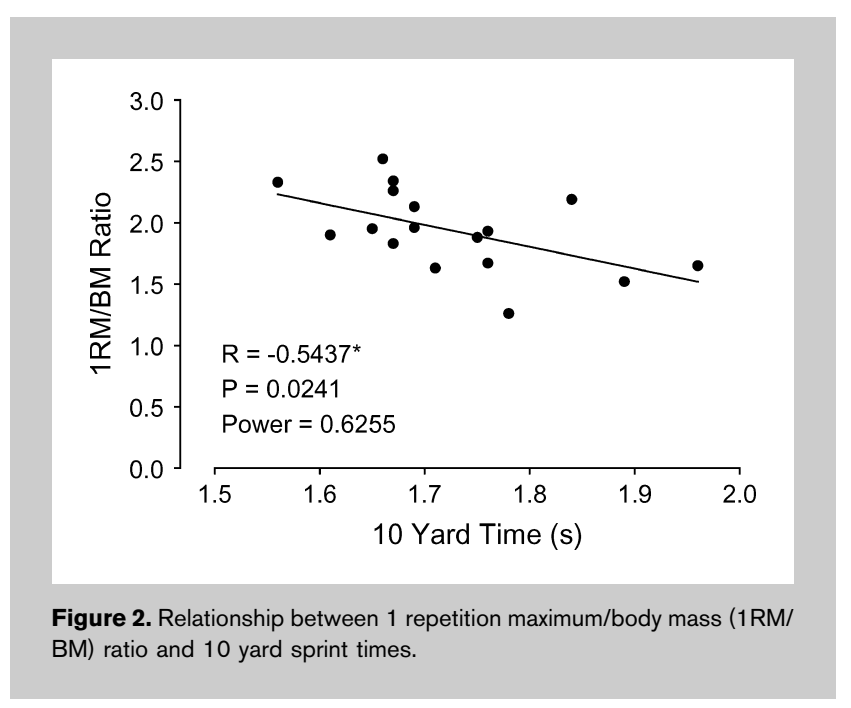


Journal of Strength and Conditioning Research $\mid$ www.nsca-jscr.org

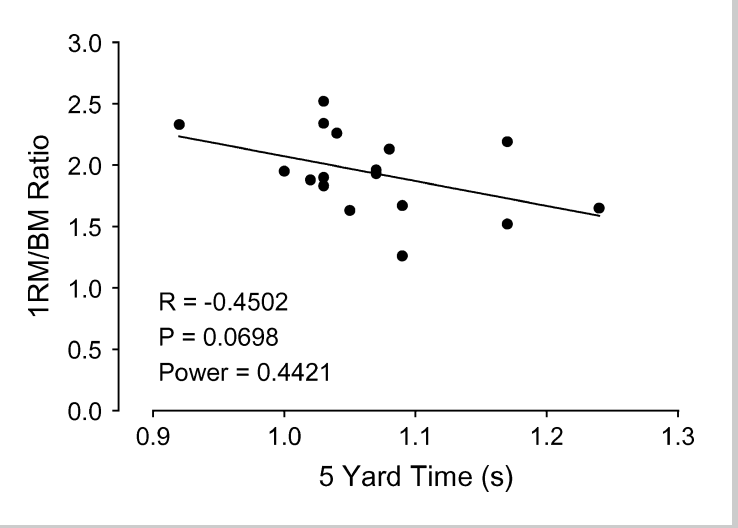

Figure 3. Relationship between 1 repetition maximum/body mass (1RM/ $\mathrm{BM})$ ratio and 5 yard sprint times.

\section{Results}

A statistically significant correlation was found between 40 yard sprint times and maximal squat $1 \mathrm{RM} / \mathrm{BM}(r=-0.6048$, power $=0.7468, p=0.0101$ ) (Figure 1 ). A statistically significant correlation was also found between 10 yard sprint times and 1RM/BM $(r=-0.5437$, power $=0.6255, p=$ 0.0241) (Figure 2). A statistically nonsignificant correlation was observed between 5 yard sprint times and 1RM/BM $(r=-0.4502$, power $=0.4421, p=0.0698)$ (Figure 3). Subjects were divided into 2 groups: (a) greater than 2.1 $1 \mathrm{RM} / \mathrm{BM}$ and (b) less than $1.91 \mathrm{RM} / \mathrm{BM}$. Subjects in group 1 had a statistically significant lower 40 yard and 10 yard sprint time in comparison with group $2(p \leq 0.05)$ (Figure 4). Sprint times between the 2 groups were not statistically significant at 5 yards.

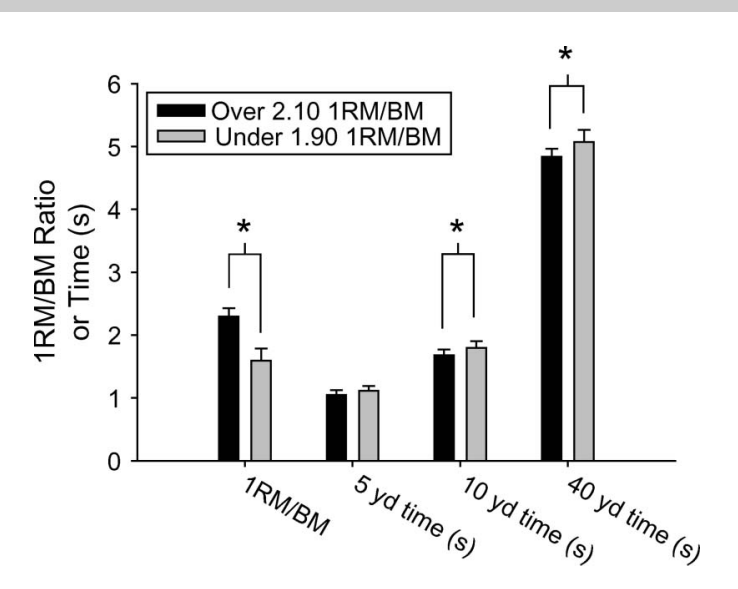

Figure 4. Mean sprint times for 40,10, and 5 yards and 1 repetition maximum/body mass (1RM/BM) ratios for group with greater than 2.1 $1 \mathrm{RM} / \mathrm{BM}$ and group with less than 1.9 1RM/BM.

\section{Discussion}

This investigation has found that $1 \mathrm{RM} / \mathrm{BM}$ is significantly related to sprinting ability in male athletes. This finding has been reported previously by Wisloff et al. (13). A relationship between ground reaction force capabilities and sprinting abilities has been previously established $(6,12)$. Thus, the strength of the lower-body musculature appears to play a role in maximal sprinting velocity. The data from this investigation further support the concept of maximizing lowerbody strength to improve sprinting ability in athletes.

Wisloff et al. (13) reported a strong statistically significant correlation of -0.940 between 10 meter sprint times and squat 1RM. The current investigation has also observed a statistically significant correlation between $1 \mathrm{RM} / \mathrm{BM}$ and 10 yard sprint times $(r=-0.5437)$. Although the correlation between strength and sprint times was not as high as previously reported by Wisloff et al. (13), the relationship was statistically significant with a relatively high power level of 0.7468 (40 yards) and 0.6255 (10 yards). Other investigations have reported much lower correlation coefficients when using singlejoint isolate measures of strength. Harris et al. (5) reported nonsignificant correlations between machine squat $1 \mathrm{RM}$ and 10 meter and 40 meter sprint times $(r=0.200,-0.140)$, and Kukolj et al. (7) reported no significant correlations between isometric peak force of knee extensors, hip extensors and hip flexors, and any sprinting performance variables.

Horizontal ground reaction force and net impulse have been strongly correlated with sprinting velocity, respectively $(r=0.7810,0.7550)(6)$. In addition, net ground reaction force has been shown to be correlated to sprinting velocity as well (12), and data also indicate that leg speed was not a factor in increased sprinting velocity (12). Thus, one of the primary factors determining sprinting velocity is the ability to generate large ground reaction forces with the lower-body musculature. Therefore, the level of strength of the lowerbody musculature is an obvious site of interest for maximizing sprinting ability.

\section{Practical Application}

In conclusion, this investigation provides additional evidence of the importance of lower-body strength in maximal sprinting performance. The amount of evidence indicating the importance of lower-body strength as assessed by a free weight structural multiple-joint measure of strength continues to increase. It is speculated that a focus of resistance training should be increasing lower-body structural multiplejoint movements of strength (i.e., free weight squat). This investigation clearly shows, in conjunction with previously reported data, that a substantial commitment to increased squat strength has a high likelihood of contributing to increased on-field sprinting ability.

\section{REFERENCES}

1. Alexander, MJ. The relationship between muscle strength and sprint kinematics in elite sprinters. Can J Sport Sci 14: 148-157, 1989. 
2. Baker, DG and Newton, RU. Comparison of lower body strength, power, acceleration, speed, agility, and sprint momentum to describe and compare playing rank among professional rugby league players. $J$ Strength Cond Res 22: 153-158, 2008.

3. Bissas, $\mathrm{AI}$ and Havenetidis, $\mathrm{K}$. The use of various strength-power tests as predictors of sprint running performance. J Sports Med Phys Fit 48: 49-54, 2008.

4. Dowson, MN, Nevill, ME, Lakomy, HK, Nevill, AM, and Hazeldine, RJ. Modelling the relationship between isokinetic muscle strength and sprint running performance. J Sport Sci 16: 257-265, 1998.

5. Harris, NK, Cronin, JB, Hopkins, WG, and Hansen, KT. Relationship between sprint times and the strength/power outputs of a machine squat jump. J Strength Cond Res 22: 691-698, 2008.

6. Hunter, JP, Marshall, RN, and McNair, PJ. Relationships between ground reaction force impulse and kinematics of sprint-running acceleration. J Appl Biomech 21: 31-43, 2005.

7. Kukolj, M, Ropret, R, Ugarkovic, D, and Jaric, S. Anthropometric, strength, and power predictors of sprinting performance. J Sports Med Phys Fit 39: 120-122, 1999.

8. McBride, JM, Triplett-McBride, T, Davie, A, and Newton, RU. The effect of heavy- vs. light-load jump squats on the development of strength, power, and speed. J Strength Cond Res 16: 75-82, 2002.
9. Smirniotou, A, Katsikas, C, Paradisis, G, Argeitaki, P, Zacharogiannis, E, and Tziortzis, S. Strength-power parameters as predictors of sprinting performance. J Sports Med Phys Fit 48: 447-454, 2008.

10. Thorstensson, A, Larsson, L, Tesch, P, and Karlsson, J. Muscle strength and fiber composition in athletes and sedentary men. Med Sci Sport 9: 26-30, 1977.

11. Weyand, PG, Lin, JE, and Bundle, MW. Sprint performanceduration relationships are set by the fractional duration of external force application. Am J Physiol 290: R758-R765, 2006.

12. Weyand, PG, Sternlight, DB, Bellizzi, MJ, and Wright, S. Faster top running speeds are achieved with greater ground forces not more rapid leg movements. J Appl Physiol 89: 1991-1999, 2000.

13. Wisloff, U, Castagna, C, Helgerud, J, Jones, R, and Hoff, J. Strong correlation of maximal squat strength with sprint performance and vertical jump height in elite soccer players. Br J Sport Med 38: 285-288, 2004.

14. Wright, $\mathrm{S}$ and Weyand, PG. The application of ground force explains the energetic cost of running backward and forward. J Exp Biol 204: 1805-1815, 2001

15. Young, W, McLean, B, and Ardagna, J. Relationship between strength qualities and sprinting performance. J Sports Med Phys Fit 35: 13-19, 1995. 